\title{
Novel roles for scleraxis in regulating adult tenocyte function
}

\author{
Anne E. C. Nichols ${ }^{1}$, Robert E. Settlage ${ }^{2}$, Stephen R. Werre ${ }^{3}$ and Linda A. Dahlgren ${ }^{1 *}$ (D)
}

\begin{abstract}
Background: Tendinopathies are common and difficult to resolve due to the formation of scar tissue that reduces the mechanical integrity of the tissue, leading to frequent reinjury. Tenocytes respond to both excessive loading and unloading by producing pro-inflammatory mediators, suggesting that these cells are actively involved in the development of tendon degeneration. The transcription factor scleraxis (Scx) is required for the development of force-transmitting tendon during development and for mechanically stimulated tenogenesis of stem cells, but its function in adult tenocytes is less well-defined. The aim of this study was to further define the role of Scx in mediating the adult tenocyte mechanoresponse.

Results: Equine tenocytes exposed to siRNA targeting Scx or a control siRNA were maintained under cyclic mechanical strain before being submitted for RNA-seq analysis. Focal adhesions and extracellular matrix-receptor interaction were among the top gene networks downregulated in Scx knockdown tenocytes. Correspondingly, tenocytes exposed to Scx siRNA were significantly softer, with longer vinculin-containing focal adhesions, and an impaired ability to migrate on soft surfaces. Other pathways affected by Scx knockdown included increased oxidative phosphorylation and diseases caused by endoplasmic reticular stress, pointing to a larger role for Scx in maintaining tenocyte homeostasis.

Conclusions: Our study identifies several novel roles for Scx in adult tenocytes, which suggest that Scx facilitates mechanosensing by regulating the expression of several mechanosensitive focal adhesion proteins. Furthermore, we identified a number of other pathways and targets affected by Scx knockdown that have the potential to elucidate the role that tenocytes may play in the development of degenerative tendinopathy.
\end{abstract}

Keywords: Tendon, Tenocyte, Scleraxis, Mechanotransduction, RNA-seq

\section{Background}

Musculoskeletal injuries are common and affect people of all ages, fitness levels, and socioeconomic groups, as well as many animal species, including horses and dogs $[1,2]$. Tendon and ligament injuries in particular account for a significant percentage of musculoskeletal injuries each year, with this number expected to rise along with an increasingly sedentary and aging population [1]. Because dense collagenous tissues such as tendon and ligament are slow to heal and the natural healing process often results in the formation of scar tissue, these injuries are particularly problematic [3]. The inability to regain normal tissue

\footnotetext{
* Correspondence: lad11@vt.edu

${ }^{1}$ Department of Large Animal Clinical Sciences, Virginia-Maryland College of Veterinary Medicine, Virginia Tech, 205 Duck Pond Drive, Blacksburg, VA 24061-0442, USA

Full list of author information is available at the end of the article
}

structure and mechanical properties often leads to tissue degeneration and chronic reinjury. Despite the frequency at which these injuries occur, and the associated loss of function and productivity they engender, there are few effective treatments available [4]. Tendinopathies are particularly difficult to treat due to their chronic and degenerative nature, which no current treatments are able to adequately resolve. This lack of treatment options is due in part to a lack of understanding of the basic biology of resident tendon cells, called tenocytes. Tenocytes are responsible for the synthesis and maintenance of the normal tendon extracellular matrix architecture in response to physiological load [5]. Decreased production of collagen and upregulation of catabolic enzymes and pro-inflammatory mediators by tenocytes in response to both excessive loading and unloading implicates these cells as a 
primary driver of degenerative tendinopathy $[6,7]$. A better understanding of how tenocytes sense and respond to physical strain could therefore lead to more effective treatments.

Much of the available information regarding tenocyte behavior has been gleaned through investigation of the basic helix-loop-helix transcription factor, scleraxis (Scx). Scx is frequently used as a tendon marker, and is critically involved in both the development of force-transmitting tendons in mice and the tenogenic differentiation of stem cells [8-10]. Mechanical load increases Scx expression $[11,12]$ and Scx is required for the pro-tenogenic effects of cyclic strain on stem cells [13]. Scx also plays a part in regulating the response to mechanical load in adult mice, with decreased expression following tendon unloading and increased expression in response to physiological load $[14,15]$. Taken together, this information demonstrates an important, but not well-characterized, role for Scx in tenocyte mechanotransduction.

To gain better insight into how Scx facilitates tenocyte mechanotransduction, we used small interfering RNA (siRNA) to knock down expression of Scx in adult equine tenocytes and subsequently exposed them to cyclic mechanical load. The resulting transcriptome was sequenced with RNA-sequencing (RNA-seq) technology and compared to that of control tenocytes. We hypothesized that Scx mediates tenocyte mechanotransduction via regulation of a specific subset of previously unidentified, mechanoresponsive genes.

\section{Methods}

\section{Tendon fibroblast isolation and culture}

For the initial transcriptome study, tenocytes were isolated from the superficial digital flexor tendon (SDFT) of a 5-year-old light breed female donated to the Virginia-Maryland College of Veterinary Medicine (VMCVM) for reasons unrelated to this study. All procedures, including tissue harvest, were performed with IACUC approval. Immediately following euthanasia by barbiturate overdose, the tensile region of both SDFT were aseptically excised, stripped of the paratenon, minced into small pieces $(2-5 \mathrm{~mm})$, and digested in growth medium (Dulbecco's modified Eagle's medium [DMEM; $4.5 \mathrm{~g} / \mathrm{L}$ glucose], 10\% FBS, $2 \mathrm{mM}$ L-glutamine, $50 \mu \mathrm{g} / \mathrm{mL}$ ascorbic acid, $25 \mathrm{mM}$ HEPES, 100 units $/ \mathrm{mL}$ penicillin, and $100 \mu \mathrm{g} / \mathrm{mL}$ streptomycin) containing 0.075\% collagenase type 2 (Worthington Biochemical, Lakewood, NJ) and $0.06 \mu \mathrm{g} / \mathrm{mL} \alpha$-ketoglutaric acid overnight at $37{ }^{\circ} \mathrm{C}, 5 \% \mathrm{CO}_{2}$, and $90 \%$ humidity. Cells were strained, pelleted, and plated at $6000 / \mathrm{cm}^{2}$ in growth medium. Growth medium was exchanged every 3 days and cells were passaged upon reaching $70 \%$ confluence using $0.25 \%$ trypsin-EDTA. For cohort validation studies, tenocytes were isolated and passaged in the same manner from the SDFT of 6 additional light breed horses (mean age $5.8 \pm 3.3$ years; 4 females, 2 castrated males) donated to the VMCVM for reasons unrelated to this study and under IACUC approval. Tenocytes were used at passage 3 for all experiments.

\section{siRNA and cyclic strain exposure}

Tenocytes were transfected (Nucleofector ${ }^{\mathrm{TN}}$ system, Lonza, Cologne, Germany) with a siRNA targeting the equine Scx mRNA (Sense: 5'-AGAGAAAGUUGAGC AAGGAtt-3', Antisense: 5'-UCCUUGCUCAACUU UCUCUgg-3', GenBank ref. NM_001105150.1; Silencer ${ }^{\text {m }}$ Select, Ambion, Life Technologies, Carlsbad, CA) or a non-targeting scramble siRNA control (Silencer ${ }^{\text {in }}$ Select Negative Control No. 1, Catalog \#4390843, Life Technologies). Transfection efficiency was evaluated using a fluorescein-conjugated scramble siRNA (sc-36,869; Santa Cruz Biotechnology Inc., Dallas, TX) and counting labeled cells by fluorescent microscopy. Cells were resuspended at $1 \times 10^{6} / 100 \mu \mathrm{L}$ in Nucleofector ${ }^{\mathrm{Tm}}$ Cell Line Solution V (Lonza) containing $10 \mathrm{nmol}$ siRNA or scramble control, transferred to cuvettes, and nucleofected using the T20 program. Cells were recovered in growth medium for $15 \mathrm{~min}$ at $37^{\circ} \mathrm{C}$ before plating at 200,000 cells/well on flexible silicone culture plates (UniFlex Collagen type I coated; Flexcell International, Hillsborough, NC). Cells were allowed to adhere for $24 \mathrm{~h}$ before being synchronized in culture medium containing $1 \%$ FBS. After $18 \mathrm{~h}$, cells were exposed to cyclic uniaxial strain $(1 \%, 0.5 \mathrm{~Hz}, 2 \mathrm{~h})$ every $24 \mathrm{~h}$ for 3 days. Thirty minutes after completion of the final strain cycle, cells were collected into guanidine isothiocyanate-phenol solution (TRIzol ${ }^{\circ}$ Reagent, Invitrogen, Carlsbad, CA) for RNA isolation. The experiment was repeated 3 times to generate 6 total samples for sequencing ( 3 siRNA and 3 scramble controls). Cohort samples for validation by qPCR were generated in the same manner.

\section{RNA isolation}

Total RNA was isolated by column purification according to manufacturer instructions (Direct-zol Microprep, Zymo Research, Irvine, VA) and evaluated both spectrophotometrically for quantity (NanoDrop, Thermo Scientific, Waltham, MA) and by electrophoresis for RNA integrity (Bioanalyzer, Agilent, Santa Clara, CA). RNA from the same samples submitted for RNA-seq and the additional cohort were isolated and converted to cDNA for use in qPCR validation studies (High-Capacity cDNA Reverse Transcription Kit, Applied Biosystems, Foster City, CA).

\section{Transcriptomic analysis}

cDNA library prep and sequencing was performed at the Biocomplexity Institute at Virginia Tech. Total RNA (1 
$\mu \mathrm{g}$ per sample) was enriched for polyA RNA (PrepX PolyA mRNA isolation kit, Wafergen, Fremont, CA) and converted to cDNA libraries (PrepX RNA-seq for Illumina Library Kit, Wafergen). Libraries underwent 13 rounds of PCR to generate the final cDNA libraries for sequencing. Individual sample libraries were clustered and sequenced on the Illumina HiSeq 2500 (average of 26.9 million paired end reads for scramble control and 29.3 million for Scx siRNA). Raw sequence data were evaluated for quality (FastQC) [16] and adaptor sequences and low quality reads were removed using Trimmomatic [17] prior to being aligned to the reference genome (EquCab2) using HISAT2 [18] and mapped to known features (Ensembl EquCab2 version 90) using HTSeq [19]. Differential gene expression between scramble control and Scx knockdown samples was determined using DESEQ2 [20]. All fold changes are shown relative to the scramble control and on a $\log 2$ scale, unless otherwise stated. Genes with a $\pm 1.5 \log 2$ fold change and an adjusted $p$-value of $p<0.05$ were used in functional annotation and gene ontology (GO) enrichment analysis of differentially expressed genes using the PANTHER Classification System (version 13.0, http://www.pantherdb.org/) and a false discovery rate of 0.05. KEGG Pathway analysis was performed with the DAVID Bioinformatics Resource (version 6.7, https://david.ncifcrf.gov/) and significance set at $p<$ 0.05 as evaluated by modified Fisher Exact test (EASE score). Sequence data generated in this study have been submitted to National Center for Biotechnology Information Gene Expression Omnibus (NCBI GEO, https://www.ncbi.nlm.nih.gov/geo/) under accession number GSE110567. Differential expression analysis and normalized counts for all genes and samples are included as Additional file 1.

\section{qPCR analysis}

Minor groove binding primer-probe sets were purchased (Scx, assay \#Ec03818452_s1, Life Technologies) or designed for genes of interest identified by the transcriptomic analysis (Primer Express ${ }^{\circ}$, Applied Biosystems; Table 1). All primer-probe sets had an efficiency of $>90 \%$ as determined by serial dilution against a known template ${\left(\text { TaqMan }^{\mathrm{Tm}}\right.}^{\text {Master Mix, Life Technologies; StepOnePlus }}{ }^{\mathrm{mm}}$
Real-Time PCR System, Applied Biosystems). Relative gene expression was calculated using the $\Delta \Delta \mathrm{Ct}$ method and the housekeeping gene glyceraldehyde 3-phosphate dehydrogenase (GAPDH) [21, 22]. Data are shown relative to the average of the scramble control samples for each gene.

\section{Immunofluorescent staining and morphological analysis}

Cells were exposed to either the scramble control or Scx-targeting siRNA and plated on collagen type I coated tissue culture polystyrene (TCP) plates in the same manner described above for cells plated on silicone bottom plates. After 3 days in culture, cells were fixed in prewarmed $4 \%$ paraformaldehyde in dPBS $+0.3 \%$ TritonX-100 for $15 \mathrm{~min}$ at room temperature. Differences in cytoskeletal and focal adhesion morphology were investigated using a focal adhesion staining kit following manufacturer's instructions (FAK100, MilleporeSigma, St. Louis, MO). Five random fields were acquired at $20 \times$ magnification per condition for each horse and used for analysis of cell morphological features (CellProfiler version 3.0.0, http://cellprofiler.org/).

\section{Migration assays}

Tenocytes were cultured on silicone bottom plates as described above, or on collagen-coated TCP. Scratches were created using a $200 \mu \mathrm{L}$ pipette tip 30 min following the end of the final strain cycle. Monolayers were rinsed once with $\mathrm{APBS}$ to remove debris and covered with fresh culture medium containing $1 \%$ FBS. Images were taken at $0,3,5,8$, and $12 \mathrm{~h}$ post scratch formation to monitor cell migration into the scratch area (ImageJ, National Institutes of Health, Bethesda, MD).

\section{Single cell stiffness measurements by atomic force microscopy}

Tenocytes were exposed to Scx siRNA and cultured on collagen-coated TCP as described above. Young's Modulus $(E)$ measurements were obtained using a Veeco BioScope II (Veeco Instruments Inc., Planview NY) equipped with a heated stage and blunted pyramidal silicon nitride cantilever tips (spring constant $=0.06 \mathrm{~N} \mathrm{~m}^{-}$ 1 , half open angle $=18^{\circ}$; DNP-10, Bruker Nano Inc., Camarillo, CA). Force-distance curves were captured in

Table 1 Equine specific primers used for $\mathrm{qPCR}$

\begin{tabular}{llll}
\hline Gene & Forward $\left(5^{\prime}\right.$ to $\left.3^{\prime}\right)$ & Reverse $\left(5^{\prime}\right.$ to $\left.3^{\prime}\right)$ & Probe $\left(5^{\prime}\right.$ to $\left.3^{\prime}\right)$ \\
\hline GAPDH & CAAGTTCCATGGCACAGTCAAG & GGCCTTCCGTTGATGACAA & CCGAGCACGGGAAG \\
BCAR1 & CCAAGATCTTTGTGGACACA & CCCGATGAACACCAGCTTGT & CAAATTCGTCATCCTCA \\
TLN1 & GAAGATGAGGCCACCAAAGG & GACCGCCAGTTCCTGACGTA & ACACGGGCCCTGGA \\
TLN2 & CCGTGTCTGACTCCATCAAGAG & TGCCATCAATGGAGTAGTCACACT & TCATCACATCTATCAGAGACAA \\
FLNB & CCTCGCTGCCACCTGATC & AGCTCCTTTGGTGTCGATGGT & TCCAAGGTGAAGGCC \\
FLNC & GGGCCAAAGGGCACAGA & ACAGGGTAGTACTCACACTCGACAC & AGCTGGTGAAGGTGCGA \\
\hline
\end{tabular}


contact mode at $1 \mathrm{~Hz}$ for a Z-scan distance of $1 \mu \mathrm{m}$. To determine Young's Modulus, raw data were fit to a modified Hertz cone model for up to $10 \%$ of the peri-nuclear cell thickness to eliminate any influence from the culture dish using Eqs. (1) and (2)

$$
\begin{aligned}
& F=k\left(d-d_{0}\right) \\
& F=\frac{2 \tan \alpha}{\pi}\left[\frac{E}{1-v^{2}}\right] \delta^{2}
\end{aligned}
$$

where $F=$ applied force, $k=$ spring constant of the cantilever, $d_{0}=$ deflection point during cell contact, $\alpha=$ half open angle of the tip, $v=$ Poisson's Ratio (0.5), and $\delta=$ indentation $[23,24]$. Triplicate force distance curves were collected and averaged for at least 8 cells per condition and repeated for each horse in the cohort $(n=7)$.

\section{Statistical analysis}

All statistical analyses were performed using SAS Studio 3.6 (SAS Institute Inc., Cary, NC). Unpaired Student's T-Tests were used to assess differences in gene expression, cell stiffness, and morphometric data. Migration assay data were analyzed by mixed model ANOVA with Tukey's post hoc testing using the PROC GLIMMIX procedure. Model fit was evaluated by examining studentized residual plots. Statistical significance was set at $p \leq 0.05$. Box and whisker plots represent the median value and 25 th and 75 th percentiles, with whiskers denoting the minimum and maximum values. All remaining data are shown as mean $\pm \mathrm{SD}$.

\section{Results}

\section{Scx knockdown by siRNA}

Transfection efficiency using the described protocol was $>95 \%$ beginning approximately $24 \mathrm{~h}$ post-nucleofection and remained $>95 \%$ until at least $96 \mathrm{~h}$. Exposure to Scx siRNA using the described protocol resulted in an average knockdown of approximately $57 \%(p<0.001)$, as measured by qPCR, in cDNA made from the same samples submitted for RNA-seq (Fig. 1a). As an initial validation of the RNA-seq data, Scx expression was compared between the two methods. The current annotation of the equine genome (EquCab2) does not include Scx as a feature, presumably due to the poor quality of the equine genome upstream of the Scx gene and incomplete information regarding the 3' end of the Scx coding region. Alignment of RNA-seq reads to the equine Scx mRNA sequence (NM_001105150.1) revealed a substantial GC bias that decreased the total number of mapped reads (Fig. 2). Nevertheless, in samples exposed to Scx siRNA, the number of reads mapping to the Scx mRNA was significantly decreased ( $p=$ 0.014) compared to the scramble control (Fig. 1b).

\section{RNA-sequencing and transcriptomic analysis}

A total of 11,166 annotated transcripts (out of 26,922) were detected in tenocytes, with 10,231 expressed in both control cells and those exposed to Scx siRNA. An additional 747 genes were expressed in only the control cells and 188 were expressed only in the Scx knockdown cells. The top 25 most highly expressed genes, regardless of Scx knockdown, are shown in Table 2. Vimentin (VIM), a fibroblast marker, was the most highly expressed gene in our dataset. The two major tendon extracellular matrix proteins, collagen types $I \alpha 2$ (COL1A2) and III 1 (COL3A1), were among the most highly expressed genes and were unaffected by Scx knockdown. The small leucine rich proteoglycans decorin (DCN) and lumican (LUM) were both highly expressed and significantly ( $p<0.001$ for both) increased in Scx-depleted tenocytes; however, the fold changes did not meet the inclusion criteria ( $\log 2$ fold change of $>/<$ 1.5) to be considered for differential expression (LUM = $1.21 \log 2$ fold change, $\mathrm{DCN}=0.72 \log 2$ fold change). To further confirm the identity of the cells as tenocytes, data were examined for expression of tendon-enriched genes [25]. Sixty out of 68 of the previously reported tendon-selective genes were present in our dataset (Table 3). The data were also parsed for changes in the expression of other tendon-related genes (Table 4).
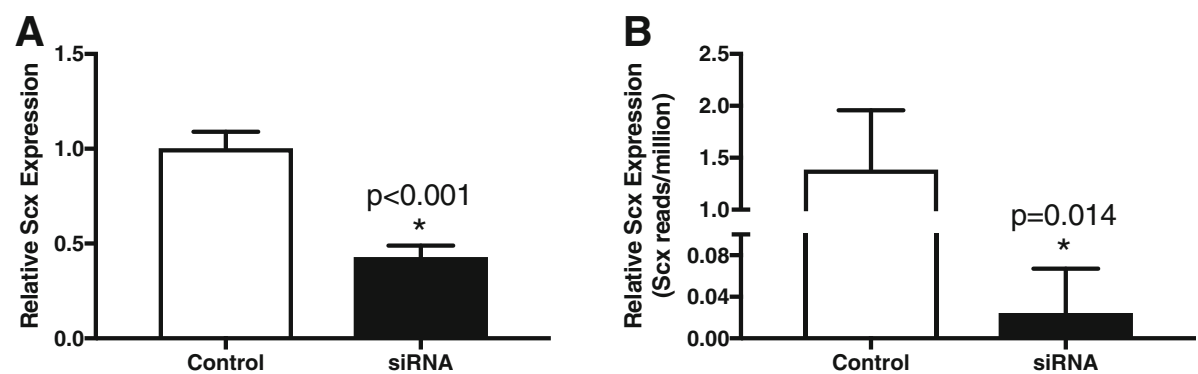

Fig. 1 Scleraxis (ScX) transcript knockdown as measured by (a) qPCR and (b) RNA-seq in sequenced samples. Equine tenocytes exposed to a siRNA targeting Scx for 3 days had decreased expression of Scx mRNA, validating both the effectiveness of the siRNA and the RNA-seq data $(n=3)$ 


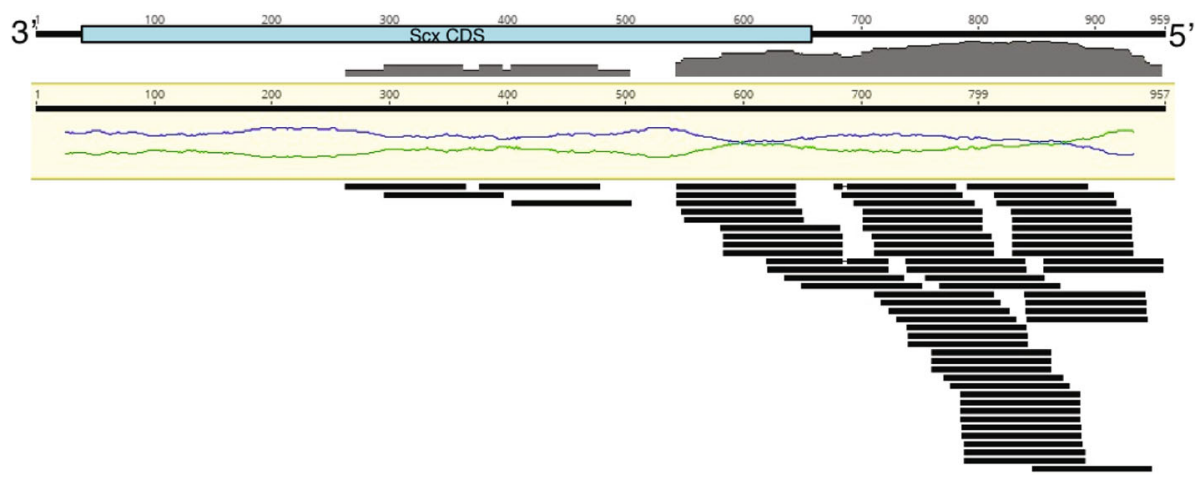

Fig. $2 \mathrm{GC}$ bias apparent in reads mapping to scleraxis $(\mathrm{Scx}) \mathrm{mRNA}$. Shown are reads from a representative scramble control mapped to the equine Scx mRNA (NM_001105150.1). GC content (blue line) of the reference sequence is shown relative to the AT content (green line). The majority of the reads mapped to the $5^{\prime}$ UTR, which has a GC content of approximately 55\%. Few reads mapped to the coding sequence (CDS; $72 \%$ GC) and those that did are preferentially located in non-GC biased regions. There were no reads that corresponded to the 3' end of the Scx CDS, which exhibited the highest GC content (approximately 80\%)

Table 2 Top 25 most highly expressed genes in equine tendon fibroblasts

\begin{tabular}{|c|c|c|c|c|c|}
\hline Gene ID & ENSEMBL Gene ID & Gene name & Base Mean & Fold Change (log2) & p-adj \\
\hline VIM & ENSECAG00000004216 & vimentin & $101,873.95$ & 0.49 & 0.0846 \\
\hline LUM & ENSECAG00000018248 & lumican & $93,834.08$ & 1.21 & 0.0000 \\
\hline EEF1A1 & ENSECAG00000020363 & $\begin{array}{l}\text { eukaryotic translation elongation } \\
\text { factor } 1 \text { alpha } 1\end{array}$ & $80,865.77$ & 0.35 & 0.4978 \\
\hline FN1 & ENSECAG00000000701 & fibronectin 1 & $76,204.43$ & -0.03 & 0.9131 \\
\hline COL3A1 & ENSECAG00000024769 & collagen type III alpha 1 & $71,782.03$ & -0.28 & 0.3120 \\
\hline COL1A2 & ENSECAG00000024740 & collagen type I alpha 2 & $68,527.63$ & 0.03 & 0.9444 \\
\hline ACTG1 & ENSECAG00000018600 & actin gamma 1 & $67,827.73$ & 0.59 & 0.1648 \\
\hline DCN & ENSECAG00000020413 & decorin & $66,957.05$ & 0.72 & 0.0000 \\
\hline CLU & ENSECAG00000007010 & clusterin & $60,592.80$ & 0.54 & 0.3689 \\
\hline CTSK & ENSECAG00000019087 & cathepsin K & $50,743.84$ & -0.01 & 0.9822 \\
\hline PSAP & ENSECAG00000021672 & prosaposin & $42,671.36$ & -0.71 & 0.0123 \\
\hline COL6A3 & ENSECAG00000020887 & collagen type $\mathrm{VI}$ alpha 3 chain & $39,357.71$ & -0.81 & 0.0000 \\
\hline AHNAK & ENSECAG00000014229 & AHNAK nucleoprotein & $32,777.16$ & -1.03 & 0.0004 \\
\hline TPT1 & ENSECAG00000018348 & tumor protein, translationally-controlled 1 & $32,069.02$ & 0.86 & 0.0000 \\
\hline RPL4 & ENSECAG00000023179 & ribosomal protein $L 4$ & $28,412.14$ & 0.55 & 0.0555 \\
\hline ANXA1 & ENSECAG00000015794 & annexin A1 & $28,358.48$ & 1.22 & 0.0000 \\
\hline HSP90AA1 & ENSECAG00000018948 & $\begin{array}{l}\text { heat shock protein } 90 \text { alpha family } \\
\text { class A member } 1\end{array}$ & $27,249.39$ & 0.36 & 0.5410 \\
\hline ASPN & ENSECAG00000007047 & asporin & $27,102.67$ & 1.44 & 0.0000 \\
\hline EIF4G2 & ENSECAG00000014700 & $\begin{array}{l}\text { eukaryotic translation initiation factor } 4 \\
\text { gamma } 2\end{array}$ & $25,563.63$ & 1.26 & 0.0000 \\
\hline HSPA8 & ENSECAG00000013510 & $\begin{array}{l}\text { heat shock protein family A (Hsp } 70) \\
\text { member } 8\end{array}$ & $23,372.98$ & -0.02 & 0.9496 \\
\hline HTRA1 & ENSECAG00000009990 & HtrA serine peptidase 1 & $23,322.81$ & -0.18 & 0.7559 \\
\hline APP & ENSECAG00000021011 & amyloid beta precursor protein & $21,959.94$ & -0.08 & 0.7687 \\
\hline CLTC & ENSECAG00000019077 & clathrin heavy chain & $21,758.80$ & 0.44 & 0.1597 \\
\hline CTNNB1 & ENSECAG00000006949 & catenin beta 1 & $21,082.51$ & 0.57 & 0.0512 \\
\hline FAP & ENSECAG00000011790 & fibroblast activation protein alpha & $20,454.17$ & 1.72 & 0.0000 \\
\hline
\end{tabular}


Table 3 Comparison of genes expressed in current dataset to previously reported tendon-selective genes

\begin{tabular}{|c|c|c|c|c|c|}
\hline Tendon Enriched Gene & Ensembl Gene ID & Mean Counts & Fold Change (log2) & p-adj & Comparison Species \\
\hline$\overline{D C N}$ & ENSECAG00000020413 & $66,957.05$ & 0.72 & $2.29 \mathrm{E}-05$ & Human \\
\hline ASPN & ENSECAG000000007047 & $27,102.67$ & 1.44 & $5.86 \mathrm{E}-07$ & Human \\
\hline THBS1 & ENSECAG00000008923 & $11,775.47$ & -0.21 & $5.86 \mathrm{E}-01$ & Human \\
\hline COL12A1 & ENSECAG000000025065 & $10,772.57$ & -0.34 & $3.85 \mathrm{E}-01$ & Rat \\
\hline PRRX1 & ENSECAG00000008539 & 3493.07 & 0.26 & 2.09E-01 & Human \\
\hline ANKRD12 & ENSECAG00000013901 & 2555.15 & 0.65 & $2.34 \mathrm{E}-01$ & Human \\
\hline FBLN1 & ENSECAG00000018101 & 2491.42 & 0.12 & $8.31 \mathrm{E}-01$ & Human \\
\hline CCL2 & ENSECAG00000023949 & 2373.29 & 1.75 & $1.20 \mathrm{E}-13$ & Rat \\
\hline ATP2B1 & ENSECAG00000008450 & 2263.22 & 0.57 & $1.18 \mathrm{E}-01$ & Rat \\
\hline PDE8A & ENSECAG00000007337 & 2224.57 & 0.36 & 2.87E-01 & Rat \\
\hline DTWD1 & ENSECAG00000012316 & 2126.12 & 1.48 & $2.68 \mathrm{E}-07$ & Rat \\
\hline BAT2D1 (PRRC2C) & ENSECAG00000016800 & 1913.66 & -0.71 & $4.85 \mathrm{E}-03$ & Human \\
\hline CREBBP & ENSECAG00000024766 & 1329.63 & -0.75 & $3.82 \mathrm{E}-02$ & Rat \\
\hline EZR & ENSECAG00000018333 & 1083.68 & -0.78 & 1.47E-02 & Human \\
\hline IL4RA & ENSECAG00000021525 & 797.67 & -1.28 & 2.49E-03 & Rat \\
\hline MKX & ENSECAG00000016778 & 797.26 & 0.25 & $5.24 \mathrm{E}-01$ & Human \\
\hline YIPF3 & ENSECAG00000016807 & 739.38 & -0.38 & $5.28 \mathrm{E}-01$ & Rat \\
\hline PSCD3 (CYTH3) & ENSECAG000000025034 & 615.61 & -0.91 & $9.38 \mathrm{E}-05$ & Rat \\
\hline COMMD7 & ENSECAG00000007694 & 546.66 & -0.01 & $9.86 \mathrm{E}-01$ & Rat \\
\hline LAMA5 & ENSECAG00000023274 & 502.79 & -1.81 & 4.36E-08 & Rat \\
\hline ARSB & ENSECAG00000020847 & 436.24 & -1.37 & $6.29 \mathrm{E}-05$ & Human \\
\hline SDC1 & ENSECAG00000014709 & 406.86 & -0.38 & $5.54 \mathrm{E}-01$ & Rat \\
\hline FNBP1 & ENSECAG00000012905 & 385.17 & -0.50 & 1.09E-01 & Rat \\
\hline GBA2 & ENSECAG00000000580 & 329.49 & -0.76 & $1.24 \mathrm{E}-01$ & Rat \\
\hline DKK3 & ENSECAG00000022804 & 317.62 & -0.50 & 3.03E-01 & Human \\
\hline RNF41 & ENSECAG00000006364 & 316.63 & -0.31 & 5.79E-01 & Rat \\
\hline LOXL4 & ENSECAG00000005573 & 310.32 & -1.18 & 9.56E-05 & Human \\
\hline MITF & ENSECAG00000005360 & 282.90 & 0.19 & $6.10 \mathrm{E}-01$ & Rat \\
\hline FBXL7 & ENSECAG00000005529 & 269.32 & -0.60 & $3.60 \mathrm{E}-01$ & Rat \\
\hline OAF & ENSECAG00000015986 & 235.41 & -1.17 & 4.05E-02 & Human \\
\hline IGFBP6 & ENSECAG00000019633 & 235.35 & -1.14 & 3.40E-02 & Human \\
\hline USF1 & ENSECAG00000004755 & 225.09 & -1.07 & $9.76 \mathrm{E}-04$ & Rat \\
\hline NOX4 & ENSECAG00000010054 & 216.23 & 0.32 & 4.77E-01 & Human \\
\hline MAB21L1 & ENSECAG00000004493 & 152.16 & -0.13 & $8.19 \mathrm{E}-01$ & Rat \\
\hline CPXM2 & ENSECAG00000024631 & 141.82 & -2.11 & $9.34 \mathrm{E}-04$ & Rat \\
\hline$X G$ & ENSECAG00000000026 & 126.06 & -1.95 & 7.34E-04 & Human \\
\hline SEMA3B & ENSECAG00000013515 & 112.02 & -0.53 & 5.97E-01 & Rat, Human \\
\hline EBF1 & ENSECAG000000007964 & 93.12 & -0.27 & $6.99 \mathrm{E}-01$ & Rat \\
\hline WNT5B & ENSECAG00000016516 & 85.46 & -2.02 & $1.30 \mathrm{E}-05$ & Rat \\
\hline ATF3 & ENSECAG00000011486 & 78.58 & 0.57 & $2.26 \mathrm{E}-01$ & Rat \\
\hline GSDMD & ENSECAG00000015005 & 73.32 & -1.81 & 1.04E-05 & Rat \\
\hline NTRK2 & ENSECAG00000011815 & 50.63 & -0.33 & $6.73 \mathrm{E}-01$ & Rat \\
\hline NOV & ENSECAG00000023039 & 40.91 & -0.24 & $8.23 \mathrm{E}-01$ & Human \\
\hline AMID (AIFM2) & ENSECAG00000004338 & 36.15 & -1.84 & 4.66E-04 & Rat \\
\hline
\end{tabular}


Table 3 Comparison of genes expressed in current dataset to previously reported tendon-selective genes (Continued)

\begin{tabular}{|c|c|c|c|c|c|}
\hline Tendon Enriched Gene & Ensembl Gene ID & Mean Counts & Fold Change (log2) & p-adj & Comparison Species \\
\hline C1QTNF2 & ENSECAG00000020786 & 19.91 & -0.72 & $3.43 \mathrm{E}-01$ & Human \\
\hline TNNI3K & ENSECAG00000010595 & 17.47 & 0.52 & 5.59E-01 & Human \\
\hline FGF18 & ENSECAG00000019045 & 17.19 & 0.17 & $8.85 \mathrm{E}-01$ & Human \\
\hline IGFBP5 & ENSECAG00000013425 & 16.46 & -2.54 & $8.23 \mathrm{E}-04$ & Rat \\
\hline THBS4 & ENSECAG00000019665 & 14.05 & -0.55 & 5.59E-01 & Rat, Human \\
\hline ELN & ENSECAG00000011106 & 9.50 & -0.74 & 4.40E-01 & Rat \\
\hline SFRP2 & ENSECAG00000017027 & 8.53 & -0.53 & $6.04 \mathrm{E}-01$ & Human \\
\hline SEPT4 & ENSECAG00000020248 & 5.80 & -0.44 & $6.59 \mathrm{E}-01$ & Rat \\
\hline KERA & ENSECAG00000017668 & 4.63 & 0.70 & 4.66E-01 & Human \\
\hline TRIM29 & ENSECAG00000013651 & 4.12 & -0.94 & $2.95 \mathrm{E}-01$ & Human \\
\hline $\operatorname{CCDC3}$ & ENSECAG00000018744 & 3.46 & -0.25 & $8.05 \mathrm{E}-01$ & Human \\
\hline FKHL18 (FOXS1) & ENSECAG00000001159 & 2.86 & -1.01 & 2.06E-01 & Rat \\
\hline DPP4 & ENSECAG00000017357 & 2.26 & 0.36 & $5.76 \mathrm{E}-01$ & Human \\
\hline MYOC & ENSECAG00000010454 & 0.16 & -0.04 & NA & Human \\
\hline GPR83 & ENSECAG00000020552 & 0.11 & -0.04 & NA & Human \\
\hline ANGPTL7 & ENSECAG00000010887 & 0.00 & NA & NA & Human \\
\hline CHODL & ENSECAG00000009963 & 0.00 & NA & NA & Human \\
\hline CNTN3 & ENSECAG00000013575 & 0.00 & NA & NA & Human \\
\hline ITIH3 & ENSECAG00000003355 & 0.00 & NA & NA & Rat \\
\hline SELE & ENSECAG00000008423 & 0.00 & NA & NA & Rat \\
\hline SERPINB7 & ENSECAG00000024951 & 0.00 & NA & NA & Rat \\
\hline TNMD & ENSECAG00000018944 & 0.00 & NA & NA & Rat, Human \\
\hline UTS2R & ENSECAG00000005300 & 0.00 & NA & NA & Rat \\
\hline
\end{tabular}

Table 4 Expression of common tendon-related genes

\begin{tabular}{|c|c|c|c|c|}
\hline Gene Name & Ensembl Gene ID & Mean Counts & Fold Change (log2) & p-adj \\
\hline$\overline{A C A N}$ & ENSECAG00000007493 & 304.22 & -2.04 & $2.40 \mathrm{E}-14$ \\
\hline BGN & ENSECAG00000018717 & 6090.64 & -0.38 & 4.74E-01 \\
\hline COL1A1 & ENSECAG00000013693 & $16,336.22$ & -1.01 & $2.78 \mathrm{E}-04$ \\
\hline COL5A1 & ENSECAG00000009361 & 1327.94 & -1.52 & 1.48E-07 \\
\hline COMP & ENSECAG00000000336 & 694.85 & -0.90 & $2.74 \mathrm{E}-03$ \\
\hline FMOD & ENSECAG00000017864 & 106.08 & -0.66 & 2.73E-01 \\
\hline MMP1 & ENSECAG00000023733 & 4.30 & -0.37 & 7.17E-01 \\
\hline MMP13 & ENSECAG00000005506 & 4779.30 & 0.85 & 2.77E-03 \\
\hline MMP2 & ENSECAG00000000953 & $10,991.67$ & -0.82 & $8.55 \mathrm{E}-03$ \\
\hline MMP3 & ENSECAG00000000750 & 72.76 & -0.49 & 5.27E-01 \\
\hline TGFB1 & ENSECAG00000011671 & 318.04 & -0.90 & 1.10E-01 \\
\hline TGFB3 & ENSECAG00000015029 & 2488.60 & -0.99 & 3.81E-04 \\
\hline TNC & ENSECAG00000017433 & 1383.05 & -0.77 & $1.99 \mathrm{E}-03$ \\
\hline
\end{tabular}

ACAN aggrecan, BGN biglycan, COL1A1 collagen type 1a1, COL5A1 collagen type 5a1, COMP cartilage oligomeric matrix protein, FMOD fibromodulin, MMP1 matrix metalloproteinase 1, MMP13 matrix metalloproteinase 13, MMP2 matrix metalloproteinase 2, MMP3 matrix metalloproteinase 3, TGFB1 transforming growth factor beta 1, TGFB3 transforming growth factor beta $3, T N C$ tenascin $C$ 
Expression of collagen types I 11 (COL1A1) and $5 \alpha 1$ (COL5A1) were decreased, as was expression of the glycoproteins tenascin-C (TNC) and cartilage oligomeric matrix protein (COMP) and the proteoglycan aggrecan (ACAN). Expression of matrix metalloproteinases (MMP) -1 and -3 was relatively low and unaffected by Scx knockdown. Conversely, MMP-13 expression was increased and MMP-2 expression was decreased in Scx-depleted cells.

Of the 11,166 annotated transcripts detected, 1002 genes met the threshold for differential expression (411 upregulated and 591 downregulated genes in Scx depleted tenocytes compared to controls). GO analysis of differentially expressed genes revealed a number of biological processes affected by Scx knockdown (Table 5). Downregulated genes exhibited significant enrichment in processes involved in cell-matrix adhesion (4.8-fold, $p$
$=0.037)$, transmembrane receptor protein tyrosine kinase signaling pathway (2.8-fold, $p=0.046$ ), cell differentiation (2.1-fold, $p=0.006)$, and developmental processes (1.6-fold, $p=0.007$ ), among others. Genes upregulated by Scx knockdown showed significant enrichment in processes including oxidative phosphorylation (6.6-fold, $p=0.02$ ), mitochondrion organization (5-fold, $p=0.009$ ), translation (3.2-fold, $p=0.011$ ), and transcription from RNA polymerase II promoter ( 1.97 -fold, $p=0.022$ ).

Differentially expressed genes were overlaid onto KEGG database pathways to further define specific pathways affected by Scx knockdown (Table 6). In agreement with the GO analysis, the extracellular matrix-receptor interaction (3.5-fold, $p=0.014$ ) and focal adhesion (2.3-fold, $p=0.017$ ) pathways were enriched in genes downregulated by Scx knockdown. Pathways represented

Table 5 Gene Ontology (GO) analysis of differentially expressed genes

\begin{tabular}{|c|c|c|c|c|}
\hline \multicolumn{2}{|c|}{ PANTHER GO-Slim Biological Process } & \multirow{2}{*}{$\frac{\text { Number of genes }}{6}$} & \multirow{2}{*}{$\begin{array}{l}\text { Fold enrichment } \\
4.79\end{array}$} & \multirow{2}{*}{$\frac{F D R}{3.7 E-02}$} \\
\hline Down-regulated & Cell-matrix adhesion (GO:0007160) & & & \\
\hline & Protein folding (GO:0006457) & 9 & 4.00 & $1.8 \mathrm{E}-02$ \\
\hline & Transmembrane receptor protein tyrosine kinase signaling (GO:0007169) & 11 & 2.77 & 4.6E-02 \\
\hline & Cytoskeleton organization (GO:0007010) & 24 & 2.33 & $6.8 \mathrm{E}-03$ \\
\hline & Cell differentiation (GO:0030154) & 30 & 2.17 & $6.1 \mathrm{E}-03$ \\
\hline & Regulation of phosphate metabolic process (GO:0019220) & 27 & 1.99 & 2.4E-02 \\
\hline & Organelle organization (GO:0006996) & 57 & 1.86 & $1.2 \mathrm{E}-03$ \\
\hline & Developmental process (GO:0032502) & 64 & 1.63 & $6.8 \mathrm{E}-03$ \\
\hline & Cellular component organization (GO:0016043) & 77 & 1.58 & $5.2 \mathrm{E}-03$ \\
\hline & Phosphate-containing compound metabolic process (GO:0006796) & 64 & 1.53 & $2.3 \mathrm{E}-02$ \\
\hline & Cellular component organization or biogenesis (GO:0071840) & 79 & 1.51 & $8.6 \mathrm{E}-03$ \\
\hline & Nitrogen compound metabolic process (GO:0006807) & 91 & 1.39 & 3.1E-02 \\
\hline & Metabolic process (GO:0008152) & 195 & 1.29 & $3.2 \mathrm{E}-03$ \\
\hline & Primary metabolic process (GO:0044238) & 153 & 1.26 & 3.7E-02 \\
\hline \multirow[t]{16}{*}{ Up-regulated } & Oxidative phosphorylation (GO:0006119) & 5 & 6.64 & 2.0E-02 \\
\hline & Mitochondrion organization (GO:0007005) & 8 & 5.00 & $8.9 \mathrm{E}-03$ \\
\hline & Protein complex biogenesis (GO:0070271) & 13 & 3.37 & $9.0 \mathrm{E}-03$ \\
\hline & Translation (GO:0006412) & 12 & 3.20 & $1.1 \mathrm{E}-02$ \\
\hline & Protein complex assembly (GO:0006461) & 12 & 3.12 & $1.2 \mathrm{E}-02$ \\
\hline & Transcription from RNA polymerase II promoter (GO:0006366) & 26 & 1.97 & $2.2 \mathrm{E}-02$ \\
\hline & Cellular component biogenesis (GO:0044085) & 26 & 1.93 & $2.3 \mathrm{E}-02$ \\
\hline & Cellular protein modification process (GO:0006464) & 26 & 1.91 & 2.4E-02 \\
\hline & Cell cycle (GO:0007049) & 23 & 1.89 & 4.5E-02 \\
\hline & Biosynthetic process (GO:0009058) & 55 & 1.78 & $3.5 \mathrm{E}-03$ \\
\hline & Organelle organization (GO:0006996) & 35 & 1.68 & 3.1E-02 \\
\hline & Protein metabolic process (GO:0019538) & 44 & 1.65 & $2.2 \mathrm{E}-02$ \\
\hline & Cellular component organization (GO:0016043) & 51 & 1.55 & $2.5 \mathrm{E}-02$ \\
\hline & Cellular component organization or biogenesis (GO:0071840) & 53 & 1.50 & 3.3E-02 \\
\hline & Primary metabolic process (GO:0044238) & 113 & 1.37 & 9.7E-03 \\
\hline & Metabolic process (GO:0008152) & 137 & 1.34 & 5.7E-03 \\
\hline
\end{tabular}


Table 6 Enrichment analysis of KEGG Pathways containing differentially expressed genes

\begin{tabular}{lllll}
\hline KEGG Pathway & & Number of genes & Fold enrichment & $p$-value \\
\hline Down & ECM-receptor interaction (ecb04512) & 7 & 3.51 & $1.35 \mathrm{E}-02$ \\
& Neurotrophin signaling pathway (ecb04722) & 8 & 2.54 & $3.55 \mathrm{E}-02$ \\
& Focal adhesion (ecb04510) & 11 & 2.34 & $1.74 \mathrm{E}-02$ \\
Up & Oxidative phosphorylation (ecb00190) & 14 & 6.38 & $1.60 \mathrm{E}-07$ \\
& Parkinson's disease (ecb05012) & 14 & 6.31 & 6.36 \\
& Ribosome (ecb03010) & 10 & 4.41 & $1.95 \mathrm{E}-07$ \\
& Alzheimer's disease (ecb05010) & 13 & 3.79 & $2.69 \mathrm{E}-05$ \\
& Huntington's disease (ecb05016) & 12 & 4.76 & $2.48 \mathrm{E}-04$ \\
& N-Glycan biosynthesis (ecb00510) & 4 & $4.93 \mathrm{E}-02$ \\
\hline
\end{tabular}

by upregulated genes included oxidative phosphorylation (6.4-fold, $p<0.001$ ), ribosome (6.4-fold, $p<0.001$ ), and several neurodegenerative diseases, including Parkinson's (6.3-fold, $p<0.001$ ), Alzheimer's (4.4-fold, $p<0.001$ ), and Huntington's disease (3.8-fold, $p<0.001)$.

\section{Validation of RNA-seq results by qPCR}

To confirm the biological significance of the transcriptomic data, the relationship between Scx knockdown and focal adhesions suggested by the GO and KEGG analyses was further explored. Several differentially expressed, focal adhesion-related genes, were chosen for validation of the sequencing data by $\mathrm{qPCR}$ in both the original samples submitted for sequencing and the additional cohort ( $n=7$ horses total). Exposure to Scx siRNA resulted in significantly decreased Scx gene expression compared to controls (Table 7; $p=0.036$ ). The remaining genes quantified by qPCR showed comparable downregulation to the RNA-seq results (Table 7), and included several adaptor proteins and those that facilitate force transduction via focal adhesion linkage to the actin cytoskeleton (talin 1 and 2 [TLN1, TLN2], filamin $\mathrm{B}$ and B [FLNB, FLNC]; Fig. 3). Other downregulated genes, including breast cancer anti-estrogen resistance protein 1 (BCAR1, also known as p130Cas) and SHC adaptor proteins 3 and 4 (SHC3, SHC4), are key players

Table 7 Comparison of gene expression fold changes in RNAseq data and $\mathrm{qPCR}$ results

\begin{tabular}{llllll}
\hline & & & \multicolumn{3}{l}{ Cohort $q P C R$} \\
\cline { 5 - 6 } \cline { 5 - 6 } Gene & RNA-seq & & Control & Scx siRNA & $p$-value \\
\hline Scx & - & & $1.52 \pm 1.36$ & $0.41 \pm 0.41$ & 0.036 \\
BCAR1 & 0.35 & & $1.07 \pm 0.45$ & $0.71 \pm 0.33$ & 0.236 \\
TLN1 & 0.33 & & $1.07 \pm 0.43$ & $0.82 \pm 0.45$ & 0.357 \\
TLN2 & 0.41 & & $1.17 \pm 0.71$ & $0.84 \pm 0.53$ & 0.105 \\
FLNB & 0.39 & & $1.05 \pm 0.35$ & $0.80 \pm 0.39$ & 0.244 \\
FLNC & 0.28 & & $1.07 \pm 0.46$ & $0.74 \pm 0.37$ & 0.231 \\
\hline
\end{tabular}

SCX scleraxis, $B C A R 1$ Breast cancer anti-estrogen resistance protein 1 (p130CAS), TLN1 Talin 1, TLN2 Talin 2, FLNB Filamin B, FLNC Filamin C in the regulation of cell migration by acting as scaffolds for tyrosine kinase-related signaling. Also downregulated in tenocytes exposed to Scx siRNA were several extracellular matrix components (collagen types V $\alpha 1$, VI $\alpha$, and IV $\alpha 2$, laminin subunits $\alpha 5$ and $\beta 2$, heparan sulfate proteoglycan 2 , and thrombospondin 2), integrin subunits $\alpha 3$ and $\beta 3$, and two regulatory subunits of protein phosphatase 1 (12B and 12C).

\section{Effects of Scx knockdown on focal adhesion morphology and cytoskeletal stiffness}

Changes in cytoskeletal and focal adhesion structure in response to Scx knockdown were examined by immunofluorescent staining for the actin cytoskeleton and vinculin, a protein found in mature focal adhesions that was unaffected by Scx knockdown (Fig. 4). No overt differences were seen in the cytoskeletal organization; however, tenocytes exposed to Scx siRNA had decreased cytosolic staining of vinculin and longer vinculin-containing focal adhesions compared to controls $(7.2 \pm 4.3$ and $4.9 \pm$ $2.6 \mu \mathrm{m}$, respectively; $p<0.001)$. In addition, tenocytes exposed to Scx siRNA were approximately $40 \%$ softer than control cells $(p<0.001)$, as measured by atomic force microscopy (Fig. 5). Scx knockdown had no significant effect on cell area or nuclear shape (Table 8).

\section{Effect of Scx knockdown on tenocyte migration}

There was no difference in cell migration between tenocytes exposed to Scx siRNA and a scramble control siRNA when cultured on collagen-coated TCP $(p=$ 0.065 ; Fig. 6). When migration assays were performed on silicone membranes, however, there was a significant two-way interaction between plate type and Scx knockdown $(p=0.025)$. Though tenocytes tended to migrate slower on the silicone membrane overall, cells exposed to Scx siRNA were significantly slower to migrate on silicone compared to TCP at $12 \mathrm{~h}$ post-scratch creation $(p<0.001$; Fig. 6). 
A

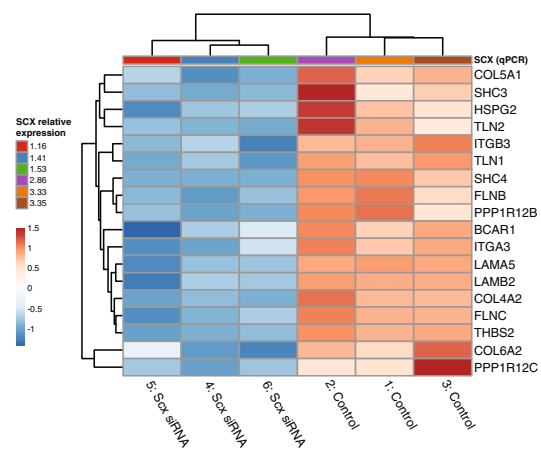

B

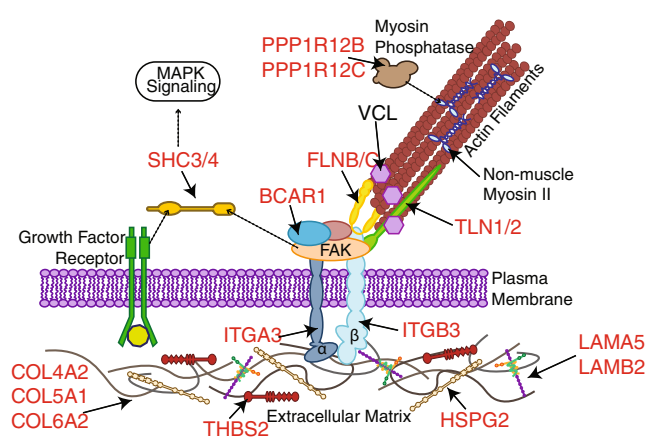

Fig. 3 Focal adhesion-related genes downregulated in tenocytes by exposure to scleraxis (Scx) siRNA. Heatmap showing normalized counts of differentially expressed genes related to focal adhesions or extracellular matrix-receptor interactions, clustered by relative Scx expression (as measured by qPCR) and expression pattern (a). Location, interactions, and downstream pathways of downregulated genes (b). Affected genes are shown in red and unaffected genes in black. Dashed lines indicate interactions between pathway components. COL4A2- collagen type IVa2, COL5A1- collagen type Va1, COL6A2- collagen type Vla2, THBS4- thrombospondin 4, ITGA3- integrin subunit alpha 3, ITGB3- integrin subunit beta 3, HSPG2- heparin sulfate proteoglycan 2, LAMA5- laminin subunit alpha 5, LAMB2- laminin subunit beta 2, TLN1- talin 1, TLN2- talin 2, FLNBfilamin B, FLNC- filamin C, BCAR1- breast cancer anti-estrogen resistance protein 1, SHC3- SHC adaptor protein 3, SHC4- SHC adaptor protein 4, PPP1R12B- protein phosphatase 1 regulatory subunit 12B, PPP1R12C-protein phosphatase 1 regulatory subunit 12C, VCL- vinculin

\section{Discussion}

By using a broad, transcriptomic approach followed by biological validation, we identified several novel Scx-mediated processes with important implications in understanding tenocyte behavior. Equine tendon fibroblasts exposed to siRNA targeting Scx were softer, showed an impaired ability to migrate on softer surfaces, and exhibited differences in focal adhesion morphology compared to controls. Together, these findings suggest a potential role and mechanism for Scx in modulating tenocyte mechanotransduction. The results of our study identify interesting new avenues for investigation into tenocyte biology that have the potential to advance our understanding of how physical cues play a role in the development of tendon disorders.

Despite the plethora of information available about the role of Scx in development, few studies have examined the role of Scx in adult tenocytes. A recent in vitro study
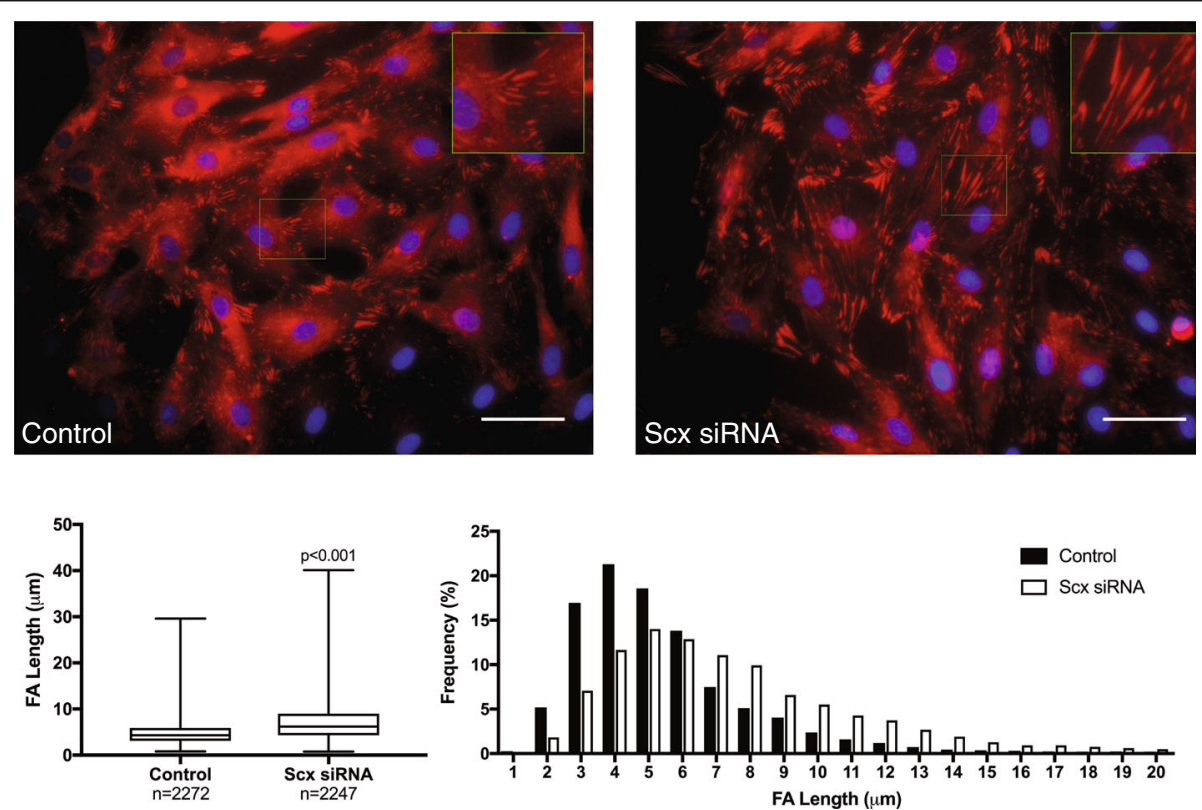

Fig. 4 Morphometric analysis of focal adhesions (FA) in tenocytes exposed to Scx siRNA compared to control tenocytes. Representative images of FA staining (top panels; red- vinculin, blue-DAPI. Scale bar $=50 \mu \mathrm{m}$ ). In cells with decreased Scx expression, FA were significantly longer those of control tenocytes (bottom panels) 


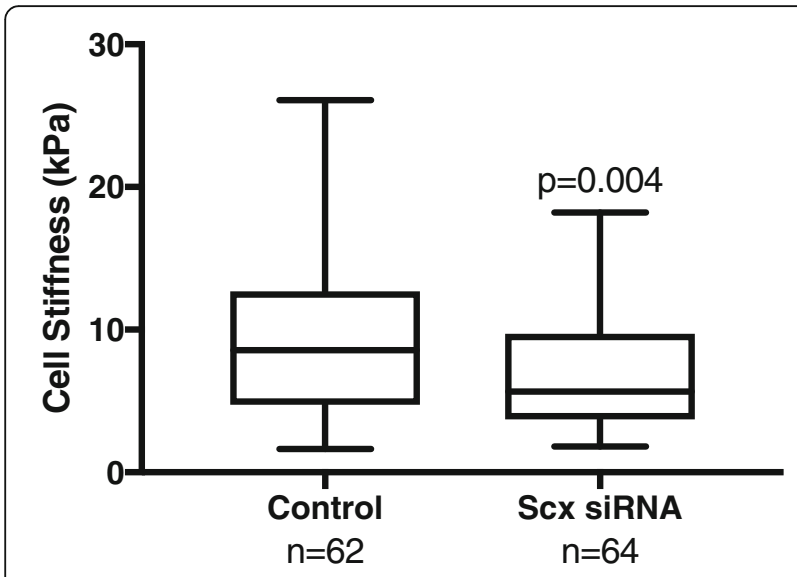

Fig. 5 Effect of Scx knockdown on tenocyte stiffness. Young's modulus for individual cells was determined by atomic force microscopy. Tenocytes exposed to Scx siRNA were significantly softer than control

demonstrated that Scx knockdown in adult equine tenocytes did not affect the expression of common tendon-related genes or the ability to reorganize a 3D collagen matrix [10]. Several groups have reported increased Scx expression in tendons following mechanical load or injury in vivo; however, it appears that the increase in Scx expression is due to proliferation of cells from the tendon periphery and their subsequent migration into the tendon core rather than increased expression by resident tenocytes themselves $[15,26,27]$. In fact, using a murine patellar defect model and Scx-GFP reporter mice, Dyment et al. reported that Scx expression in tenocytes located in the tendon core adjacent to the injured area sharply decreased following injury and remained decreased for at least 7 days before slowly recovering [26]. This suggests that there are at least two Scx-positive cell populations involved in tendon repair and remodeling, though their contributions to overall Scx expression need further clarification. In our study, care was taken to remove the paratenon/epitenon prior to cell isolation in order to examine the effects of Scx knockdown in tenocytes from the tendon parenchyma specifically, though we cannot rule out the presence of endotenon-derived cells.

Scx expression is frequently used as a marker of tenocyte identity, but it is also expressed in other tissues [28, 29]. Tenomodulin (TNMD) and thrombospondin 4

Table 8 Cell area and nucleus shape of tenocytes exposed to SCX SiRNA and control

\begin{tabular}{llll}
\hline & Nuclear Eccentricity & Cell Area $\left(\mu \mathrm{m}^{2}\right)$ & $n$ \\
\hline Control & $0.63 \pm 0.12$ & $2183.81 \pm 592.02$ & 1623 \\
Scx siRNA & $0.63 \pm 0.13$ & $2153.26 \pm 284.23$ & 1447 \\
$p$-value & 0.85 & 0.06 & \\
\hline
\end{tabular}

(THBS4) are enriched in tendon compared to other tissues in humans and rats [25]; however, the use of TNMD as a marker of tenocyte identity in horses is less supported, as similar levels of TNMD are found in both tendon and bone [30] and at least one study was unable to detect TNMD expression in normal equine SDFT [31]. In our dataset generated from passaged tenocytes, we observed low levels of THBS4 expression and undetectable levels of TNMD. Despite this, many other tendon selective genes were expressed. Interestingly, a number of these genes, including asporin (ASPN), C-C Motif Chemokine Ligand 2 (CCL2), Laminin Subunit Alpha 5 (LAMA5), and Wnt Family Member 5B (WNT5B), were significantly affected by Scx knockdown. These findings suggest that Scx plays an active role in promoting adult tenocyte identity.

Exposure to Scx siRNA resulted in significantly decreased Scx mRNA as measured by both RNA-seq and qPCR. Scx is not included in the current version (EquCab2) of the equine genome annotation. Therefore, to evaluate Scx expression in our dataset, we mapped sample reads against the Scx mRNA. It is important to note that Scx mRNA is relatively small (957 bp containing two exons), GC-rich ( 70\% overall), and contains regions of stark GC content disparity, ranging from 50 to $80 \%$. As a result, coverage across the Scx mRNA was reduced. GC bias between samples and genes in RNA-seq data are well-documented effects and there are numerous tools to account for these biases in analysis pipelines [32, 33]. Within-transcript bias is also relatively common, but correcting for it is less defined. Use of GC unaware transcript estimation methods can lead to errors in transcript abundance, especially when examining differential isoform expression [34]. In the case of Scx, there are no documented transcript variants; however, care should be taken in evaluating the expression of Scx in transcriptomic studies, as low levels of expression may relate to bias in the sequencing technology and not a biological phenomenon.

Functional annotation and GO analysis showed that Scx knockdown significantly downregulated pathways involved in focal adhesion and extracellular matrix-receptor interactions in our study. Focal adhesions are large protein complexes that form the physical link connecting the cytoskeleton to the extracellular matrix through the binding and activation of transmembrane proteins called integrins [35]. Integrins themselves are direct mechanosensors, and upon activation facilitate the recruitment of many cytosolic proteins to the plasma membrane to form the intracellular portion of the adhesion complex [36]. The protein talin, in particular, anchors the actin cytoskeleton to the focal adhesion by force-dependent interaction with the cytosolic tail of the $\beta 3$ integrin (ITGB3) [37, 38]. Talin is a critical mechanosensor, and loss of talin impairs cell migration and extracellular substrate sensing [39, 40]. 


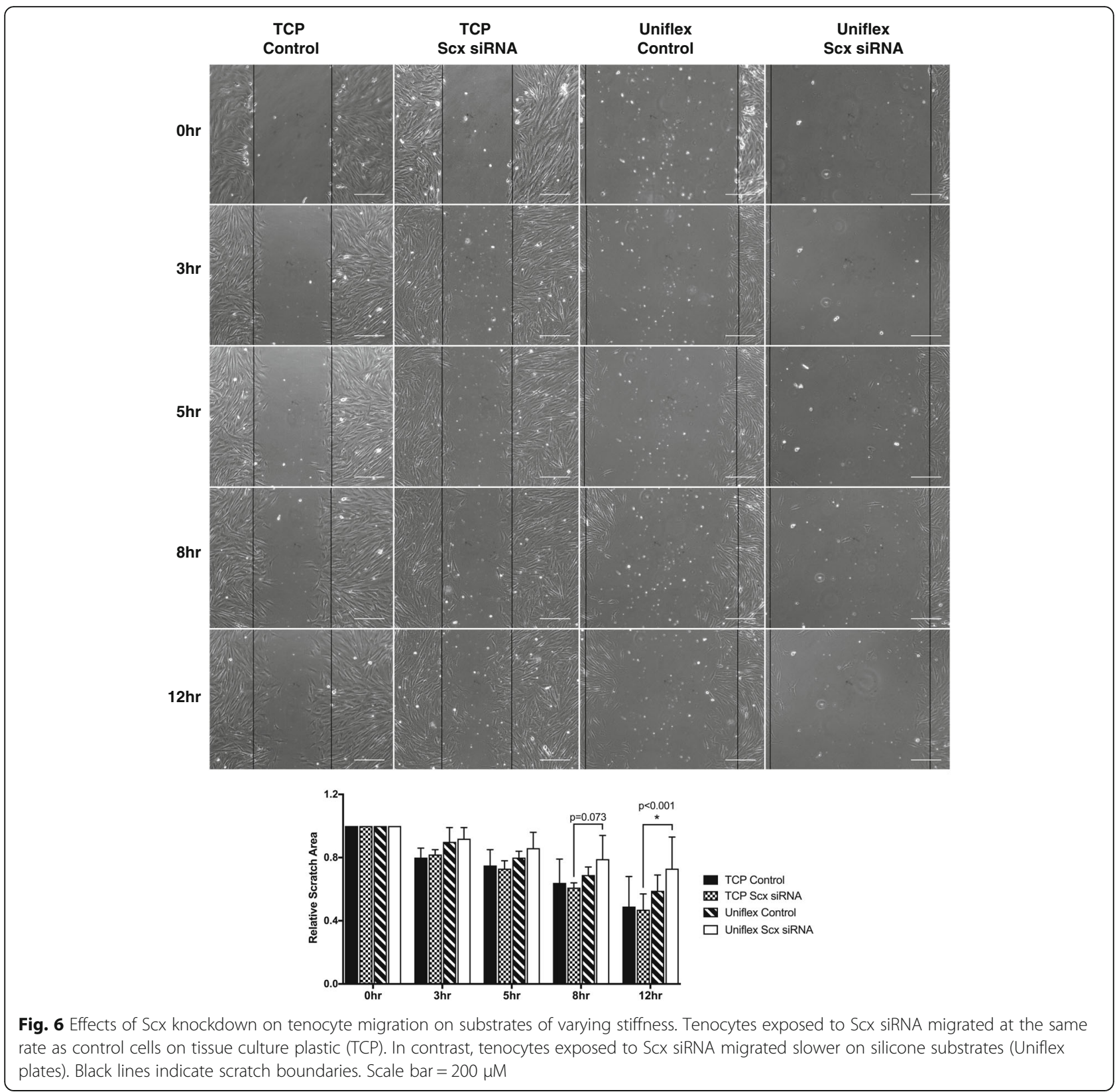

Following talin activation, the cytosolic protein vinculin is recruited to the focal adhesion and interacts with talin to stabilize the complex [41]. Increased presence of vinculin is indicative of decreased focal adhesion turnover and more mature adhesion, which inhibits cell migration [41, 42].

Other proteins recruited in response to integrin binding, including BCAR1 and the SHC adaptor proteins, enable the integration of physical and chemical cues into downstream pathway activation in response to mechanical stimulation. Increased BCAR1 expression correlates with increased invasive potential of cancer cells, and silencing of BCAR1 or SHC3/4 results in decreased migratory ability [43-46]. Expression of both talin isoforms (TLN1 and TLN2), ITGB3, BCAR1, and SHC3/4 were decreased in tenocytes following Scx knockdown in our study. Consistently, tenocytes exposed to Scx siRNA had longer vinculin-containing focal adhesions, indicating decreased focal adhesion turnover. Despite the presence of longer focal adhesions, there was no effect of Scx knockdown on tenocyte migration on TCP. Interestingly, Scx-depleted tenocytes migrated more slowly on softer silicone membranes, whereas control cells were unaffected by the change in substrate stiffness. As many of the genes affected by Scx knockdown function as link proteins at the interface between integrins and the actin 
cytoskeleton, this substrate stiffness-dependent migration effect could reflect an inability to generate proper cytoskeletal traction.

In further support of a role for Scx in modulating cytoskeletal tension, tenocytes exposed to Scx siRNA exhibited a significant decrease in cytoskeletal stiffness compared to controls. Previous studies have reported that cells alter their cytoskeletal tension in response to environmental changes in order to maintain a predetermined tensional homeostasis [47, 48]. Loss of tensional homeostasis in tenocytes leads to upregulation of MMP-13 and downregulation of COL1A1 $[49,50]$. Recovery of cytoskeletal tension in tenocytes occurs through actin-mediated interaction with the local environment [51]. In our study, we observed a similar increase in MMP-13 and decrease in COL1A1 expression, in addition to reduced cytoskeletal stiffness. Inability of the tenocytes to form adequate focal adhesion to actin cytoskeleton connections due to decreased expression of the key adaptor proteins seen in our study (i.e. TLN1/2, FLNB/B, SHC3/4) would impair ability of tenocytes to sense and respond to changes in substrate stiffness and could therefore result in reduced cytoskeletal stiffness.

The relationship between cell stiffness and migratory capacity varies between cell type and disease state. In cancer, decreased cytoskeletal stiffness can be used as an accurate measure of metastatic potential, with cancerous cells being softer than the surrounding healthy cells [52, 53]. In non-cancerous cells, the relationship is less clear. A study by Kole et al. found that in normal 3 T3 fibroblasts, non-migratory cells were significantly softer than migrating cells, indicating that an increase in cytoskeletal stiffness is a prerequisite for directed cell migration [54]. Other studies have shown that disturbed cytoskeletal architecture or connections results in cells with decreased cytoskeletal stiffness and migratory capacity [55, 56]. We observed similar cell behavior in our study, with softer tenocytes migrating more slowly than their stiffer counterparts on softer surfaces. Substrate stiffness, cytoskeletal tension, and migratory capacity are inextricably linked in all cell types. Future studies in tenocytes will help to elucidate the specific relationships and mechanisms involved.

We validated the transcriptomic data by showing that Scx knockdown resulted in slower migrating, softer tenocytes; however, the pathway analysis points to a broader role for $\mathrm{Scx}$ in tenocyte homeostasis. Intriguingly, GO term and KEGG pathway analysis showed that a significant number of genes upregulated in response to Scx knockdown are involved in pathways related to neurodegenerative diseases (e.g., Alzheimer's, Huntington's, and Parkinson's). These particular diseases develop, in part, due to dysregulation of the unfolded protein response (UPR), a homeostatic mechanism that has evolved to counter endoplasmic reticular (ER) stress as a result of misfolded proteins [57]. Correspondingly, GO term analysis showed upregulation of many anabolic processes and a concurrent decrease in genes related to protein folding in Scx-depleted cells. ER stress and the UPR are implicated in development of organ fibrosis in heart, lung, and liver disease [58-60]. As the development of fibrotic scar tissue is a major consequence of tendon injury and the main reason for reinjury, this suggested connection between Scx and ER stress in tenocytes warrants further investigation.

\section{Conclusions}

This study is the first to identify specific roles for Scx in adult tenocytes by exposure to siRNA targeting Scx and subsequent RNA-seq interrogation. We confirmed the biological significance of the transcriptomic data by demonstrating that Scx knockdown results in the formation of abnormal focal adhesions, decreased cytoskeletal stiffness, and an impaired ability to migrate on soft substrates. Our data suggest that Scx facilitates tenocyte mechanosensing in part by regulating the expression of several focal adhesion components and genes involved in maintaining cytoskeletal tension. Whether this is the result of direct or indirect gene regulation by Scx remains to be clarified. We also identified other genes and pathways affected by Scx knockdown that point to a larger role for Scx in maintaining adult tenocyte homeostasis. Further exploration of these novel Scx-mediated targets has the potential to advance our understanding of how mechanical strain can lead to tendon injuries.

\section{Additional file}

Additional file 1: Transcriptomic Data. Differential expression analysis and normalized counts for all genes and samples. (XLSX $2345 \mathrm{~kb}$ )

\footnotetext{
Abbreviations

ACAN: Aggrecan; AMID (AIFM2): Apoptosis Inducing Factor, Mitochondria Associated 2; ANGPTL7: Angiopoietin Like 7; ANKRD12: Ankyrin Repeat Domain 12; ARSB: Arylsultatase B; ASPN: Asporin; ATF3: Activating Transcription Factor 3; ATP2B1: ATPase Plasma Membrane $\mathrm{Ca}^{2+}$ Transporting 1; BAT2D1 (PRRC2C): Proline Rich Coiled-Coil 2C; BCAR1: Breast Cancer AntiEstrogen Resistance Protein 1; C1QTNF2: C1q And TNF Related 2; CCDC3: Coiled-Coil Domain Containing 3; CCL2: C-C Motif Chemokine Ligand 2; CHODL: Chondrolectin; CNTN3: Contactin 3; COL12A1: Collagen Type XII Alpha 1; COL1A1: Collagen Type I Alpha I; COL4A2: Collagen Type IV Alpha 2; COL5A1: Collagen Type V Alpha 1; COL6A2: Collagen Type VI Alpha 2; COMMD7: COMM Domain Containing 7; COMP: Cartilage Oligomeric Matrix Protein; CPXM2: Carboxypeptidase X, M14 Family Member 2; CREBBP: CREB Binding Protein; DCN: Decorin; DKK3: Dickkopf-related Protein 3; DPP4: Dipeptidyl Peptidase 4; DTWD1: DTW Domain Containing 1;

EBF1: Early B-cell Factor 1; ELN: Elastin; EZR: Ezrin; FBLN1: Fibulin 1; FBXL7: Fbox and Leucine Rich Repeat Protein 7; FGF18: Fibroblast Growth Factor 18; FKHL18 (FOXS1): Forkhead Box S1; FLNB: Filamin B; FLNC: Filamin C; FNBP1: Formin Binding Protein 1; GAPDH: Glyceraldehyde 3-Phosphate Dehydrogenase; GBA2: Flucosylceramidase Beta 2; GO: Gene Ontology; GPR83: G Protein-Coupled Receptor 83; GSDMD: Gasdermin-D; HSPG2: Heparin Sulfate Proteoglycan 2; IGFBP5: Insulin Like Growth Factor
} 
Binding Protein 5; IGFBP6: Insulin-like Growth Factor Binding Protein 6; IL4RA: Interleukin-4 Receptor Alpha; ITGA3: Integrin Subunit Alpha 3; ITGB3: Integrin Subunit Beta 3; ITIH3: Inter-Alpha-Trypsin Inhibitor Heavy Chain 3; KERA: Keratocan; LAMA5: Laminin Subunit Alpha 5; LAMB2: Laminin Subunit Beta 2; LOXL4: Lysyl Oxidase Like 4; MAB21L1: Mab-21 Like 1; MITF: Melanogenesis Associated Transcription Factor; MKX: Homeobox Protein Mohawk; MMP: Matrix Metalloproteinase; MYOC: Myocilin; NOV: Nephroblastoma Overexpressed; NOX4: NADPH Oxidase 4; NTRK2: Neurotrophic Receptor Tyrosine Kinase 2; OAF: Out At First Homolog; PDE8A: Phosphodiesterase 8A; PPP1R12B: Protein Phosphatase 1 Regulatory Subunit 12B; PPP1R12C: Protein Phosphatase 1 Regulatory Subunit 12C: PRRX1: Paired Related Homeobox 1; PSCD3 (CYTH3): Cytohesin-3; RNAseq: RNA-sequencing; RNF41: Ring Finger Protein 41; Scx: Scleraxis; SDC1: Syndecan 1; SDFT: Superficial digital flexor tendon; SELE: Selectin E; SEMA3B: Semaphorin 3B; SEPT4: Septin 4; SERPINB7: Serpin Family B Member 7; SFRP2: Secreted Frizzled Related Protein 2; SHC3: SHC Adaptor Protein 3; SHC4: SHC Adaptor Protein 4; siRNA: small-interfering RNA; TCP: Tissue Culture Polystyrene; THBS1: Thrombospondin 1; THBS2: Thrombospondin 2; THBS4: T; rombospondin 4; TLN1: Talin 1; TLN2: Talin 2; TNC: Tenascin C; TNMD: Tenomodulin; TNNI3K: TNNI3 Interacting Kinase; TRIM29: Tripartite Motif Containing 29; UPR: unfolded protein response; USF1: Upstream Stimulatory Factor 1; UTS2R: Urotensin 2 Receptor; VCL: Vinculin; WNT5B: Wnt Family Member 5B; XG: Xg Blood Group; YIPF3: Yip1 Domain Family Member

\section{Acknowledgements}

The authors thank Andrew Ford for his invaluable assistance and guidance in the atomic force microscopy studies and Dr. Roderick Jensen for the helpful discussions regarding transcriptomic analysis.

\section{Funding}

This work was supported by internal funding from the VMCVM. AECN received graduate fellowship support from the Stamps Family Charitable Foundation. Publication costs for this article were supported by the Virginia Tech Open Access Subvention Fund.

\section{Availability of data and materials}

Data supporting the results of this study are included as Additional file 1 and are available under accession number GSE1 10567 at the National Center for Biotechnology Information Gene Expression Omnibus (NCBI GEO, https:// www.ncbi.nlm.nih.gov/geo/)

\section{Authors' contributions}

AECN and LAD conceived the study and design with assistance from RES; experiments were performed by AECN; data were analyzed by AECN, LAD, and SRW; the manuscript was written and edited by AECN and LAD. All authors read and approved the final manuscript.

\section{Ethics approval and consent to participate}

Collection of equine tissues for cell isolation was approved by the Virginia Tech IACUC under protocols 14-128 and 15-059.

\section{Consent for publication}

Not applicable

\section{Competing interests}

The authors declare that they have no competing interests.

\section{Publisher's Note}

Springer Nature remains neutral with regard to jurisdictional claims in published maps and institutional affiliations.

\section{Author details}

'Department of Large Animal Clinical Sciences, Virginia-Maryland College of Veterinary Medicine, Virginia Tech, 205 Duck Pond Drive, Blacksburg, VA 24061-0442, USA. ${ }^{2}$ Advanced Research Computing, Virginia Biocomplexity Institute, Virginia Tech, Blacksburg, VA 24061, USA. ${ }^{3}$ Laboratory for Study Design and Statistical Analysis, Virginia-Maryland College of Veterinary Medicine, Blacksburg, VA 24061, USA.
Received: 2 March 2018 Accepted: 30 July 2018

Published online: 07 August 2018

\section{References}

1. Yelin E, Weinstein S, King T. The burden of musculoskeletal diseases in the United States. Semin Arthritis Rheum. 2016;46(3):259-60.

2. Clegg PD. Musculoskeletal disease and injury, now and in the future. Part 2: tendon and ligament injuries. Equine Vet J. 2012;44(3):371-5.

3. Yang $G$, Rothrauff BB, Tuan RS. Tendon and ligament regeneration and repair: clinical relevance and developmental paradigm. Birth Defects Res C Embryo Today. 2013;99(3):203-22.

4. Goldin M, Malanga GA. Tendinopathy: a review of the pathophysiology and evidence for treatment. Phys Sportsmed. 2013:41(3):36-49.

5. Birch HL. Tendon matrix composition and turnover in relation to functional requirements. Int J Exp Pathol. 2007;88(4):241-8.

6. Xu Y, Murrell GAC. The basic science of tendinopathy. Clin Orthop Relat Res. 2008;466(7):1528-38.

7. Gardner K, Arnoczky SP, Caballero O, Lavagnino M. The effect of stressdeprivation and cyclic loading on the TIMP/MMP ratio in tendon cells: an in vitro experimental study. Disabil Rehabil. 2008;30(20-22):1523-9.

8. Schweitzer R, Chyung JH, Murtaugh LC, Brent AE, Rosen V, Olson EN, et al. Analysis of the tendon cell fate using Scleraxis, a specific marker for tendons and ligaments. Development. 2001;128(19):3855-66.

9. Murchison ND, Price BA, Conner DA, Keene DR, Olson EN, Tabin CJ, et al. Regulation of tendon differentiation by scleraxis distinguishes forcetransmitting tendons from muscle-anchoring tendons. Development. 2007; 134(14):2697-708

10. Bavin EP, Atkinson F, Barsby T, Guest DJ. Scleraxis is essential for tendon differentiation by equine embryonic stem cells and in equine fetal tenocytes. Stem Cells Dev. 2017;26(6):441-50.

11. Scott A, Danielson P, Abraham T, Fong G, Sampaio AV, Underhill TM. Mechanical force modulates scleraxis expression in bioartificial tendons. J Musculoskelet Neuronal Interact. 2011;11(2):124-32.

12. Kuo CK, Tuan RS. Mechanoactive tenogenic differentiation of human mesenchymal stem cells. Tissue Eng Part A. 2008;14(10):1615-27.

13. Li Y, Ramcharan M, Zhou Z, Leong DJ, Akinbiyi T, Majeska RJ, et al. The role of Scleraxis in fate determination of mesenchymal stem cells for tenocyte differentiation. Sci Rep. 2015;5:13149.

14. Maeda T, Sakabe T, Sunaga A, Sakai K, Rivera Alexander L, Keene Douglas R, et al. Conversion of mechanical force into TGF- $\beta$-mediated biochemical signals. Curr Biol. 2011;21(11):933-41.

15. Mendias CL, Gumucio JP, Bakhurin KI, Lynch EB, Brooks SV. Physiological loading of tendons induces scleraxis expression in epitenon fibroblasts. J Orthop Res. 2012;30(4):606-12

16. Andrews S. FastQC: a quality control tool for high throughput sequence data. Available online at: [http://www.bioinformatics.babraham.ac.uk/ projects/fastqc/]. Accessed 2 Aug 2018.

17. Bolger AM, Lohse M, Usadel B. Trimmomatic: a flexible trimmer for Illumina sequence data. Bioinformatics. 2014;30(15):2114-20.

18. Kim D, Langmead B, Salzberg SL. HISAT: a fast spliced aligner with low memory requirements. Nat Methods. 2015;12(4):357-60.

19. Anders S, Pyl PT, Huber W. HTSeq-a Python framework to work with highthroughput sequencing data. Bioinformatics. 2015;31(2):166-9.

20. Love Ml, Huber W, Anders S. Moderated estimation of fold change and dispersion for RNA-seq data with DESeq2. Genome Biol. 2014;15(12):550.

21. Livak KJ, Schmittgen TD. Analysis of relative gene expression data using real-time quantitative PCR and the 2(-Delta Delta $C(T))$ method. Methods. 2001;25(4):402-8.

22. Cardwell RD, Dahlgren LA, Goldstein AS. Electrospun fibre diameter, not alignment, affects mesenchymal stem cell differentiation into the tendon/ligament lineage. J Tissue Eng Regen Med. 2014;8(12):937-45.

23. Ford AJ, Jain G, Rajagopalan P. Designing a fibrotic microenvironment to investigate changes in human liver sinusoidal endothelial cell function. Acta Biomater. 2015;24:220-7.

24. Thomas G, Burnham NA, Camesano TA, Wen Q. Measuring the mechanical properties of living cells using atomic force microscopy. J Vis Exp. 2013;76

25. Jelinsky SA, Archambault J, Li L, Seeherman H. Tendon-selective genes identified from rat and human musculoskeletal tissues. J Orthop Res. 2010;28(3):289-97. 
26. Dyment NA, Liu CF, Kazemi N, Aschbacher-Smith LE, Kenter K, Breidenbach AP, et al. The paratenon contributes to scleraxis-expressing cells during patellar tendon healing. PLoS One. 2013;8(3):e59944.

27. Scott A, Sampaio A, Abraham T, Duronio C, Underhill TM. Scleraxis expression is coordinately regulated in a murine model of patellar tendon injury. J Orthop Res. 2011;29(2):289-96.

28. Muir T, Sadler-Riggleman I, Skinner MK. Role of the basic helix-loop-helix transcription factor, scleraxis, in the regulation of Sertoli cell function and differentiation. Mol Endocrinol. 2005;19(8):2164-74.

29. Levay AK, Peacock JD, Lu Y, Koch M, Hinton RB, Kadler KE, et al. Scleraxis is required for cell lineage differentiation and extracellular matrix remodeling during murine heart valve formation in vivo. Circ Res. 2008;103(9):948-56.

30. Taylor SE, Vaughan-Thomas A, Clements DN, Pinchbeck G, Macrory LC, Smith RKW, et al. Gene expression markers of tendon fibroblasts in normal and diseased tissue compared to monolayer and three dimensional culture systems. BMC Musculoskelet Disord. 2009;10:27.

31. Watts $A E$, Nixon AJ, Yeager AE, Mohammed HO. A collagenase gel/physical defect model for controlled induction of superficial digital flexor tendonitis. Equine Vet J. 2012;44(5):576-86.

32. Benjamini Y, Speed TP. Summarizing and correcting the GC content bias in high-throughput sequencing. Nucleic Acids Res. 2012;40(10):e72.

33. Risso D, Schwartz K, Sherlock G, Dudoit S. GC-content normalization for RNA-Seq data. BMC Bioinform. 2011;12:480.

34. Love MI, Hogenesch JB. Modeling of RNA-seq fragment sequence bias reduces systematic errors in transcript abundance estimation. Nat Biotechnol. 2016;34(12):1287-91.

35. Ross TD, Coon BG, Yun S, Baeyens N, Tanaka K, Ouyang M, et al. Integrins in Mechanotransduction. Curr Opin Cell Biol. 2013;25(5):613-8.

36. Campbell ID, Humphries MJ. Integrin structure, activation, and interactions. Cold Spring Harb Perspect Biol. 2011;3(3)

37. Patil S, Jedsadayanmata A, Wencel-Drake JD, Wang W, Knezevic I, Lam SC. Identification of a Talin-binding site in the integrin beta(3) subunit distinct from the NPLY regulatory motif of post-ligand binding functions. The Talin n-terminal head domain interacts with the membrane-proximal region of the beta(3) cytoplasmic tail. J Biol Chem. 1999;274(40):28575-83.

38. Yan J, Yao M, Goult BT, Sheetz MP. Talin dependent Mechanosensitivity of cell focal adhesions. Cell Mol Bioeng. 2015;8(1):151-9.

39. Rahikainen R, von Essen M, Schaefer M, Qi L, Azizi L, Kelly C. Mechanical stability of Talin rod controls cell migration and substrate sensing. Sci Rep. 2017:7(1):3571.

40. Austen K, Ringer P, Mehlich A, Chrostek-Grashoff A, Kluger C, Klingner C, et al. Extracellular rigidity sensing by Talin isoform-specific mechanical linkages. Nat Cell Biol. 2015;17(12):1597-606.

41. Humphries JD, Wang P, Streuli C, Geiger B, Humphries MJ, Ballestrem C. Vinculin controls focal adhesion formation by direct interactions with Talin and actin. J Cell Biol. 2007;179(5):1043-57.

42. Saunders RM, Holt MR, Jennings L, Sutton DH, Barsukov IL, Bobkov A, et al. Role of vinculin in regulating focal adhesion turnover. Eur J Cell Biol. 2006; 85(6):487-500

43. Cunningham-Edmondson AC, Hanks SK. p130Cas substrate domain signaling promotes migration, invasion, and survival of estrogen receptornegative breast cancer cells. Breast Cancer (Dove Med Press). 2009;1:39-52.

44. Honda H, Nakamoto T, Sakai R, Hirai H. p130(Cas), an assembling molecule of actin filaments, promotes cell movement, cell migration, and cell spreading in fibroblasts. Biochem Biophys Res Commun. 1999;262(1):25-30.

45. Ortensi B, Osti D, Pellegatta S, Pisati F, Brescia P, Fornasari L, et al. Rai is a new regulator of neural progenitor migration and glioblastoma invasion. Stem Cells. 2012;30(5):817-32

46. Fagiani E, Giardina G, Luzi L, Cesaroni M, Quarto M, Capra M, et al. RaLP, a new member of the Src homology and collagen family, regulates cell migration and tumor growth of metastatic melanomas. Cancer Res. 2007; 67(7):3064-73.

47. Peyton SR, Ghajar CM, Khatiwala CB, Putnam AJ. The emergence of ECM mechanics and cytoskeletal tension as important regulators of cell function. Cell Biochem Biophys. 2007:47(2):300-20.

48. Solon J, Levental I, Sengupta K, Georges PC, Janmey PA. Fibroblast adaptation and stiffness matching to soft elastic substrates. Biophys J. 2007; 93(12):4453-61.

49. Arnoczky SP, Lavagnino M, Egerbacher M, Caballero O, Gardner K, Shender MA. Loss of homeostatic strain alters Mechanostat "set point" of tendon cells in vitro. Clin Orthop Relat Res. 2008;466(7):1583-91.
50. Lavagnino M, Arnoczky SP. In vitro alterations in cytoskeletal tensional homeostasis control gene expression in tendon cells. J Orthop Res. 2005; 23(5):1211-8.

51. Gardner K, Lavagnino M, Egerbacher M, Arnoczky SP. Re-establishment of cytoskeletal tensional homeostasis in lax tendons occurs through an actinmediated cellular contraction of the extracellular matrix. J Orthop Res. 2012; 30(11):1695-701.

52. Xu W, Mezencev R, Kim B, Wang L, McDonald J, Sulchek T. Cell stiffness is a biomarker of the metastatic potential of ovarian cancer cells. PLoS One. 2012;7(10):e46609.

53. Beliveau A, Thomas G, Gong J, Wen Q, Jain A. Aligned Nanotopography promotes a migratory state in glioblastoma Multiforme tumor cells. Sci Rep. 2016;6:26143.

54. Kole TP, Tseng Y, Jiang I, Katz JL, Wirtz D. Intracellular mechanics of migrating fibroblasts. Mol Biol Cell. 2005;16(1):328-38.

55. Lee JS, Hale CM, Panorchan P, Khatau SB, George JP, Tseng Y, et al. Nuclear Lamin a/C deficiency induces defects in cell mechanics, polarization, and migration. Biophys J. 2007;93(7):2542-52.

56. Deng S, Huang C. E3 ubiquitin ligases in regulating stress fiber, lamellipodium, and focal adhesion dynamics. Cell Adhes Migr. 2014;8(1):49-54.

57. Bravo R, Parra V, Gatica D, Rodriguez AE, Torrealba N, Paredes F, et al. Endoplasmic reticulum and the unfolded protein response: dynamics and metabolic integration. Int Rev Cell Mol Biol. 2013;301:215-90.

58. Groenendyk J, Lee D, Jung J, Dyck JRB, Lopaschuk GD, Agellon LB, et al. Inhibition of the unfolded protein response mechanism prevents cardiac fibrosis. PLoS One. 2016;11(7):e0159682

59. Marcinak SJ, Ron D. The unfolded protein response in lung disease. Proc Am Thorac Soc. 2010;7(6):356-62.

60. Malhi H, Kaufman RJ. Endoplasmic reticulum stress in liver disease. J Hepatol. 2011:54(4):795-809.

Ready to submit your research? Choose BMC and benefit from:

- fast, convenient online submission

- thorough peer review by experienced researchers in your field

- rapid publication on acceptance

- support for research data, including large and complex data types

- gold Open Access which fosters wider collaboration and increased citations

- maximum visibility for your research: over 100M website views per year

At BMC, research is always in progress.

Learn more biomedcentral.com/submissions 